\title{
Erfahrungen mit der schweizerischen Entschuldungsfazilität
}

\section{Marco Ferroni et Roger Denzer}

\section{(2) OpenEdition}

1 Journals

Édition électronique

URL : http://journals.openedition.org/sjep/1437

DOI : 10.4000/sjep. 1437

ISSN : 1663-9677

Éditeur

Institut de hautes études internationales et du développement

\section{Édition imprimée}

Date de publication : 1 janvier 1994

Pagination : 295-297

ISSN : $1660-5926$

\section{Référence électronique}

Marco Ferroni et Roger Denzer, «Erfahrungen mit der schweizerischen Entschuldungsfazilität »,

Schweizerisches Jahrbuch für Entwicklungspolitik [En ligne], 13 | 1994, mis en ligne le 10 juin 2013,

consulté le 08 septembre 2020. URL : http://journals.openedition.org/sjep/1437 ; DOI : https://doi.org/ 10.4000/sjep.1437 


\section{Erfahrungen mit der schweizerischen Entschuldungsfazilität}

Marco Ferroni / Roger Denzer1

Der Artikel von Kappel zeichnet sich durch seine Ausgewogenheit aus; wir teilen die Analyse Kappels weitgehend. Der vorliegende Kommentar versteht sich als Ergänzung zum genannten Artikel und als vorläufige Aufarbeitung gewisser Erfahrungen mit den bisher durchgeführten Entschuldungsaktionen der Schweiz.

Es sei in Erinnerung gerufen, dass der Bund im Rahmen seiner Entschuldungsfazilität bisher verschiedene Rückkaufaktionen von Schulden getätigt oder unterstützt hat (auf bilateraler Ebene wurden Forderungen schweizerischer Gläubiger in der Höhe von 1,4 Mrd SFr erworben). 1993 wurde mit acht Schuldnerländern über den Erlass dieser Forderungen verhandelt. Was ist der Nutzen dieses Schuldenabbaus?

Die Beantwortung dieser Frage setzt eine Unterscheidung nach kurzfristigen und längerfristigen Auswirkungen voraus. Schuldenerlasse können einen positiven Einfluss auf die Kreditbedingungen bei kurzfristigen Handelskrediten ausüben, da die Banken diese unter anderem in Funktion der Höhe der ausstehenden Schulden festlegen. Andere messbare Effekte des Wegfalls des (mehrheitlich) nicht geleisteten Schuldendienstes werden normalerweise erst mittel- oder längerfristig wirksam (z.B. das Erreichen einer tragbaren Schuldenbelastung im Verhältnis mit den Wachstumsmöglichkeiten).

Von grosser Bedeutung für das Schuldnerland scheinen aber die Demonstrationseffekte der Schuldenreduktion zu sein. Die bisherigen Verhandlungen mit den Schuldnerländern in Lateinamerika, Afrika und dem Mittleren Osten haben gezeigt, dass dem schweizerischen Abkommen wegen seinem Pilotcharakter positive Externalitäten zugesprochen werden. Man erwartet davon, dass andere bilaterale Gläubiger sich zu analogen Aktionen des Schuldenabbaus ermuntern lassen.

In analoger Weise zu verfahren, hiesse erstens unter angemessenen Bedingungen Schuldenreduktionen von $100 \%$ auf allen ausstehenden Fälligkeiten zu gewähren. Bis heute wandeln die meisten bilateralen Kreditoren im Rahmen der Bestimmungen des Pariser Klubs nur maximal 10\% der ausstehenden Schulden in Lokalwährung um. Die Diskussion in verschiedenen internationalen Foren, an der sich auch die Schweiz beteiligt, deutet darauf hin, dass unter anderem im Pariser Klub weitergehende Schuldenerleichterungen in den Bereich des Möglichen gelangen. So bestehen bereits Vorschläge von anderen Ländern (Belgien, Grossbritannien und Nordiker), welche der Idee des Erlasses von Kapitalforderungen ("debt stock reduction") das Wort reden. In diesem Zusammenhang wird in

1. Die vorliegenden Ausführungen stellen die persönliche Meinung der Autoren dar, welche sich nicht unbedingt mit derjenigen ihrer Institution zu decken braucht. 
zunehmendem Masse auf die diesbezügliche Politik der Schweiz verwiesen.

Der Pilotcharakter der schweizerischen Abkommen besteht zweitens in der bescheidenen Umwandlungsrate der Aussenschuld in Lokalwährung. Die meisten bilateralen Gläubiger haben bis anhin Umwandlungsraten zwischen $50 \%$ und $100 \%$ gefordert. Diese hohen Raten stellen eine enorme Belastung des Budgets des Schuldnerlandes dar (aus der Sicht des Budgets betrachtet, findet de facto eine Umschuldung zu schlechteren als die entsprechenden in den Pariser KlubAbkommen vorgesehenen Bedingungen statt). Mit ihren relativ bescheidenen Umwandlungsraten tragen die schweizerischen Entschuldungsabkommen der Budgetbelastung des Schuldners Rechnung. Auf alle Fälle zu vermeiden ist ein Druck zur inflationären Entschuldung durch übermässige Geldschöpfung zur Finanzierung der Gegenwertfonds und die Gefährdung makroökonomischer Zielvorgaben, welche mit dem IWF vereinbart wurden. Viele Gläubiger und Schuldner anerkennen heute, dass bei vergangenen Konversionen von Bankschulden zu hohe Umwandlungsraten angewendet worden sind.

Die Schweiz legt die Umwandlungsrate generell so fest, dass sie zwischen den kurzfristigen, effektiven Ersparnissen der schweizerischen Entschuldung und dem Realwert der Schulden - welcher sich am Marktpreis orientiert - zu liegen kommt. Verschiedene Kriterien, wie die Beurteilung der Entwicklungs- und Budgetpolitik bzw. der Wille zu wirtschaftspolitischen Reformen, die Absorptionsfähigkeit im Bereich zusätzlicher Entwicklungsprojekte, sowie die Verschuldungssituation des Landes werden bei der Festlegung der Konversionsrate berücksichtigt. Die bis heute verhandelten Umwandlungsraten liegen in einer Spanne von $8 \%$ bis $27 \%$. Mit Nicaragua wurde aufgrund des geringen Schuldenvolumens und dessen schwacher Zahlungskapazität in Lokalwährung kein Gegenwertfonds vereinbart. Die Verhandlungsergebnisse zeigen, dass die Schuldnerländer trotz verschiedener Einwände bereit sind, einen über den kurzfristigen, effektiven Ersparnissen liegenden Lokalwährungsbetrag zur Verfügung zu stellen. Diese Differenz wird vom Schuldnerland explizit als "Preis" für das Abkommen bzw. die Schuldenreduktion interpretiert.

Mit Blick auf andere bilaterale Gläubiger spielt die Schweiz mit inrem Entschuldungsprogramm also eine Vorreiterrolle in Bezug auf Schuldenerlasse und -umwandlungen. Es ist zu früh, um beurteilen zu können, ob diese Rolle eine katalytische Wirkung haben wird, in dem Sinne, dass sie mithilft, das Problem der Überschuldung vieler ärmerer Entwicklungsländer auf breiter Front einer Lösung zuzuführen. In einem oder zwei Jahren wird man zu dieser Frage mehr wissen.

In der Zwischenzeit fallen die Entwicklungseffekte der schweizerischen Entschuldungsabkommen an. Diese umfassen erstens Devisenersparnisse von jährlich 15 bis 20 Mio SFr, welche ohne Entschuldung von den bis heute durch Schuldenrückkäufe begünstigten Ländern effektiv an schweizerische Gläubiger bezahlt worden wären und zweitens die Realisierung von mindestens teilweise zusätzlichen Entwicklungsprojekten zugunsten bedürftiger Bevölkerungsschichten. Diese Projekte werden aus den Gegenwertfonds finanziert.

Die Schwerpunkte der Verhandlungen im Bereich der Gegenwertfonds sind neben der bereits angesprochenen Frage der Konversionsrate die Anlagepolitik 
des Fonds, die sektorielle Zuweisung der Mittel und die Trägerschaft der Projekte. Die Anlagepolitik muss folgenden Anforderungen genügen: die Mittel müssen marktgerecht verzinst werden (zumindest Inflationsschutz) und sie müssen gemäss den Auszahlungsbedürfnissen der damit finanzierten Projekte verfügbar sein (Sicherheitsbedürfnis). Diese Anforderungen rufen normalerweise nach einer Anlage in einer Privatbank, womit gleichzeitig der inländische Finanzsektor gestützt werden kann.

In den meisten Fällen werden die Mittel also aus dem Regierungsbudget herausgenommen - ein Vorgehen, das grundsätzlich (das heisst aus budgetpolitischen Überlegungen heraus) nicht über alle Zweifel erhaben ist, sich aber aus folgenden Gründen aufdrängt und rechtfertigt: geringe Umwandlungsrate und das Prinzip der schweizerischen Entschuldungsfazilität, wonach der Nutzen der Entschuldung auch einheimischen bedürftigen Bevölkerungsschichten direkt zuzukommen hat. In Ländern, in denen die Budgetpolitik des Staates dies noch nicht genügend gewährleistet, sind spezielle Programme in der Art der entschuldungsinduzierten Gegenwertfonds gerechtfertigt. In dem Ausmasse wie die Projekte von staatlichen Trägern durchgeführt werden, fliessen die Mittel - nach Entscheid des für die Verwendung des Gegenwertfonds verantwortlichen Komitees - natürlich de facto wieder an die Staatskasse zurück.

Die Sektorallokation und die Identifikation der Projekt-Trägerschaft wird in jedem Land individuell festgelegt. Armutsbekämpfung, Umwelterhaltung und Kleinkreditprogramme sind immer wiederkehrende Themen. Die Trägerschaft setzt sich normalerweie aus Regierungsorganisationen, Nicht-Regierungsorganisationen und multilateralen Agenturen zusammen. Die ersten über Gegenwertfonds finanzierten Programme sind angelaufen. Der in Bolivien im April 1993 geäufnete Fonds ist bereits vollständig für Projekte verpflichtet. Die Auszahlung der Mittel dürfte zwei bis drei Jahre in Anspruch nehmen. 